\title{
A Lagrange Multiplier-based Technique within the Nonlinear Finite Element Method in Cracked Columns
}

\author{
Kaveh Salmalian ${ }^{1}$, Ali Alijani ${ }^{{ }^{*}}$, Habib Ramezannejad Azarboni² \\ ${ }^{1}$ Department of Mechanical Engineering, Bandar Anzali Branch, Islamic Azad University, 43131 Bandar Anzali, Iran \\ 2 Department of Mechanical Engineering, Ramsar Branch, Islamic Azad University, Ramsar, Iran \\ * Corresponding author, e-mail: alijani@iaubanz.ac.ir
}

Received: 06 May 2020, Accepted: 03 September 2020, Published online: 13 October 2020

\begin{abstract}
In this research, two energy-based techniques, called Lagrange multiplier and conversion matrix, are applied to involve crack parameters into the non-linear finite element relations of Euler-Bernoulli beams made of functionally graded materials. The two techniques, which divide a cracked element into three parts, are implemented to enrich the secant and tangent stiffness matrices. The Lagrange multiplier technique is originally proposed according to the establishment of a modified total potential energy equation by adding continuity conditions equations of the crack point. The limitation of the conversion matrix in involving the relevant non-linear equations is the main motivation in representing the Lagrange multiplier. The presented Lagrange multiplier is a problem-solving technique in the cracked structures, where both geometrical nonlinearity and material inhomogeneity areas are considered in the analysis like the post-buckling problem of cracked functionally graded material columns. Accordingly, some case-studies regarding the post-buckling analysis of cracked functionally graded material columns under mechanical and thermal loads are used to evaluate the results.
\end{abstract}

Keywords

Lagrange multiplier, cracked column, post-buckling, finite element

\section{Introduction}

The numerical methods -especially the finite element method (FEM)- are extensively used to evaluate the stability of cracked structures. The modeling of the crack in the framework of the FEM is carried out through different tools like the rotational spring, discrete cracked element, enriched element, etc. The concepts of the stress intensity factor, the energy release rate, and the crack opening displacement are commonly used to analyze problems including the crack. An effective tool to incorporate cracks in structures, which was firstly applied by Irwin [1], is the rotational spring model. Ricci and Viola [2] developed a theoretical method to compute the stress intensity factors of cracked Timoshenko beams. The vibration of the EulerBernoulli beam including a single crack was investigated by using a modified line-spring model in [3]. Static and dynamic analysis of cracked concrete beams was experimentally performed using the FEM by Mazaheri et al. [4]. Okamura et al. [5] investigated the buckling of cracked columns by extracting the relationship between the stress intensity factor and compliance. Skrinar [6] carried out the bending, free vibration and buckling analysis of beams by using FEM and a simplified crack model. Moreover, Biondi and Caddemi [7] investigated uniform Euler-Bernoulli beams with discontinuities. They modeled discontinuities as singularities of the flexural stiffness. The XFEM as a powerful and reliable method is successfully utilized in the analysis of cracked structures. A relevant research work can be found in [8].

Functionally graded materials (FGMs) are special composites in which the material properties change from one side to another continuously. These materials have extensive usage in engineering applications especially in aerospace, electronics, and biomedical industry. Thermal buckling analysis of functionally graded Euler-Bernoulli beams with temperature-dependent properties was investigated by Chen et al. [9]. Thermal buckling of functionally graded piezoelectric Timoshenko beams with different boundary conditions was theoretically investigated by Nasirzadeh et al. [10]. Darvizeh et al. [11] carried out the mechanical and thermal post-buckling analyses for the FGM Euler-Bernoulli beams. The nonlinear static response of FGM beams was investigated through an exact solution 
based on shear deformation theory in [12]. The non-linear thermal buckling analysis of FGM Timoshenko beams under non-uniform temperature distribution was studied by Paul and Das [13] in which the effect of the volume fraction and length-thickness ratio on the post-buckling behavior was evaluated.

Due to the significant role of the crack in reducing the stability of structures, it is important to investigate the buckling and post-buckling behaviors of cracked FGM structures. The elastic buckling of Timoshenko FGM beams including open edge crack modeled by the elastic rotational spring was studied through an analytical approach by Ke et al. [14]. Song et al. [15] investigated the thermal buckling and post-buckling of edge-cracked functionally graded multilayer graphene nanocomposite beams on an elastic foundation. The post-buckling analysis of bi-directional functionally graded imperfect beams was performed using GDQM and Newton-Raphson iteration based on a novel third-order shear deformation theory in [16].

One of the proper techniques to satisfy boundary and continuity conditions is Lagrange Multiplier (LM) which is successfully used in fracture and contact mechanics. Abraham and Brandon [17] utilized sub-structure normal modes in the vibration analysis of cantilever beams including the transverse crack. They employed the LM technique to enforce the continuity conditions in the cracked zone. Bordas et al. [18] represented a three-dimensional meshfree method to investigate the initiation, propagation, and growth of cracks. They used the extrinsic enrichment approach and LM technique in the static and dynamic analysis of non-linear materials. Sun et al. [19] developed the multi-scale lattice method for the mesoscopic crack growth simulation of concrete structures. Here Lagrange multipliers $\lambda$ is used to connect the trans-scale boundary between macro-scale and meso-scale regions. Moreover, Bruno et al. [20] proposed the LM technique to analyze mixed-mode delamination of laminated composites based on fracture and contact mechanics. Liu et al. [21] applied the LM technique to ensure the compatibility on the interface of crack tip region and outer region without crack. Luciano et al. [22] proposed variational formulations to solve the problem of bending and buckling of Timoshenko nano-beams in which Lagrange multipliers were used in the numerical analyses.

A novel technique, which was recently represented to derive the stiffness matrix of the crack element, is called the Conversion Matrix (CM) in which the tangent and secant stiffness matrices are enriched through the crack properties.
Alijani et al. [23] introduced the CM technique in the static analysis of cracked Euler-Bernoulli beams resting on an elastic foundation. Moreover, a one-dimensional finite element model using the CM technique was proposed to investigate the nonlinear behavior of cracked beams in [24].

The principal objective of this research is to present a new LM technique in the FE solution of cracked FGM column by considering the Euler-Bernoulli beam theory and the rotational spring model. The LM technique is originally implemented for the mechanical and thermal post-buckling analysis. Moreover, The CM technique which has been already introduced to involve the crack in the beam, see [25], is briefly reviewed. The main difference between the two techniques is highlighted where the geometrical nonlinearity and material inhomogeneity should be considered in the analysis. The limitation in the CM leads to neglecting the nonlinear terms and inhomogeneity in the continuity equations, while this deficiency has been remedied in the LM. Some case studies in the post-buckling analysis are used to investigate the results of two techniques. The Influence of crack depth, end conditions, load types, and material properties of the cracked FGM column is also investigated.

\section{Problem formulation}

A technique into the framework of the one-dimensional FEM is introduced to analyze the time-independent problems of open-edge cracked FGM beams. This technique is implemented based on the LM technique to analyze the non-linear buckling. Moreover, a review regarding the CM technique is presented to obtain the post-buckling path. The modeling of the crack in the FGM column, Linearization, stiffness matrix, and loading conditions are explained in-details for these two techniques.

\subsection{Modeling of crack}

Fig. 1 shows a cracked FGM column with $L, h, b$ as length, height, and width, respectively. The longitudinal axis which passes through the centroid of the cross-section is considered $x$ and Young's modulus changes in $\mathrm{z}$ direction according to exponential law as follows:

$$
E(z)=E_{0} e^{\beta z},
$$

in which $E_{0}$ and $\beta$ are Young's modulus at mid-plane $(z=0)$ and constant relevant to gradual variation of material properties, respectively. In this study, the material properties are assumed at room temperature and temperatureindependent [26]. FGM column is made from two materials 


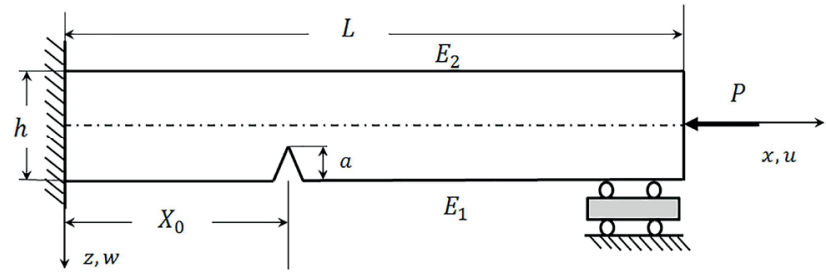

(a)

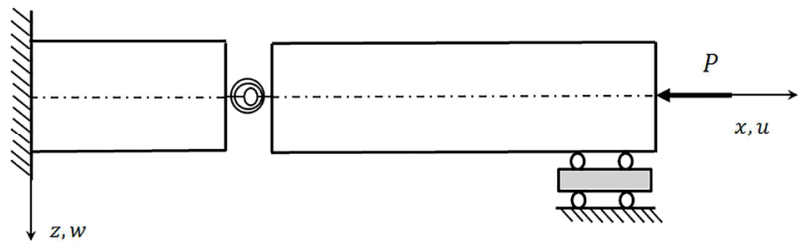

(b)

Fig. 1 Schematic description for crack modeling of metal-ceramic FGM beam under axial loading: a) open edge crack, b) modeling of crack with rotational spring

with index notations of "1" and "2" in which the crack is incorporated in the hand side of the index "1" shown in Fig. 1(a). A rotational spring is used to model the crack located in $X_{0}$ position illustrated in Fig. 1(b). The rotational spring stiffness factor is realized by the material and geometric characteristics of the crack.

The stiffness factor of the rotational spring, $K_{t}$ is obtained by calculating spring flexibility factor $\hat{G}$ as follows

$K_{t}=1 / \hat{G}$

The flexibility factor $G$ which corresponds to the crack parameter can be found from Broek's approximation [27] as

$\frac{\left(1-v^{2}\right) k_{I}^{2}}{E(a)}=\frac{M^{2}}{2} \frac{d \hat{G}}{d a}$,

where $M$ is the bending moment at the cracked section, $k_{I}$ is the stress intensity factor (SIF) associated with the opening mode $I$ and $E$ is Young's modulus at the crack tip [28]. The stress intensity factor $k_{I}$ can be computed from the data represented by Erdogan and $\mathrm{Wu}$ [29] as

$k_{I}=\frac{6 M \sqrt{\pi h \xi}}{h^{2}} F(\xi)$,

in which the crack depth ratio is $\xi=a / h$ and $\xi \leq 0.7$. Also, the expressions of $F(\xi)$ are calculated based on the mentioned results in [30]. Those have been specified for the different ratios of $E_{2 /} E_{1}=0.2,1$ and 5 as in Eq. (5) [28], Eq. (6) and Eq. (7), respectively

$$
\begin{aligned}
F(\xi) & =1177.980 \xi^{7}-2409.170 \xi^{6}+1947.830 \xi^{5} \\
& -770.54 \xi^{4}+146.776 \xi^{3}-4.742 \xi^{2}-2.752 \xi+1.91
\end{aligned}
$$

$$
\begin{aligned}
F(\xi) & =-1031.750 \xi^{7}+2395.830 \xi^{6}-2124.310 \xi^{5} \\
& \mp 909.375 \xi^{4}-192.451 \xi^{3}+21.667 \xi^{2}-1.662 \xi+1.15 \\
F(\xi) & =-211.706 \xi^{7}+545.139 \xi^{6}-535.236 \xi^{5} \\
& +267.910 \xi^{4}-72.627 \xi^{3}+12.511 \xi^{2}-0.859 \xi+0.65
\end{aligned}
$$

Substituting Eq. (4) into Eq. (3) results in the determination of the flexibility factor as in Eq. (8) [14]

$\hat{G}=\int_{0}^{\bar{\xi}} \frac{72 \pi\left(1-v^{2}\right) \xi F^{2}(\xi)}{E(\xi h) h^{2}} d \xi$

in which $\bar{\xi}$ is the given crack depth ratio.

\subsection{Non-linear finite element analysis}

One-dimensional elements are utilized to discretize the column for the finite element analysis. Moreover, longitudinal and transverse displacements in an element are interpolated by using Lagrangian and Hermitian shape functions, respectively, based on nodal displacements. Secant and tangent stiffness matrices are derived by inserting kinematic and constitutive relations into the total potential energy equation.

$\Pi=U+\Omega$,

in which, $\Pi, U$ and $\Omega$ are the total potential energy of column, the strain energy and the potential energy of external forces, respectively. Two mechanical and thermal forces are separately applied to the FGM column in which forces are considered as incremental loadings in order to solve the post-buckling problem.

\subsubsection{FE analysis for FGM columns}

The kinematic and constitutive equations of the EulerBernoulli beam are, respectively.

$\varepsilon_{x}=\varepsilon_{0}+\varepsilon_{N L}$

$\sigma_{x}=\sigma_{0}+\sigma_{N L}=E(z) \varepsilon_{x}$

Young's modulus in the FGM beam is considered as a function of $z$ direction based on Eq. (1). A common form of the kinematic equation in the FE analysis is

$\varepsilon_{0}=\frac{\partial u}{\partial x}-z \frac{\partial^{2} w}{\partial x^{2}}=\left(\boldsymbol{B}_{u}-z \boldsymbol{B}_{b}\right) \boldsymbol{u}=\boldsymbol{B}_{0} \boldsymbol{u}$

$\varepsilon_{N L}=\frac{1}{2}\left(\frac{\partial w}{\partial x}\right)^{2}=\frac{1}{2} \tilde{A} \theta=\frac{1}{2} \boldsymbol{B}_{N L} \boldsymbol{u}$. 
The tangent stiffness matrix is obtained as [30]

$\boldsymbol{K}_{T}=\boldsymbol{K}_{0}+\boldsymbol{N}_{1}+\boldsymbol{N}_{2}$,

in which $\boldsymbol{K}_{0}, \boldsymbol{N}_{1}$, and $\boldsymbol{N}_{2}$ are the matrices of independent, linearly dependent, and quadratically dependent upon the displacement vector, respectively. Those can be computed for the FGM beams as

$$
\begin{aligned}
& \boldsymbol{K}_{0}=b \int_{0}^{l_{e}}\left(\begin{array}{c}
\hat{A} \boldsymbol{B}_{\boldsymbol{u}}^{T} \boldsymbol{B}_{\boldsymbol{u}}+\hat{D} \boldsymbol{B}_{\boldsymbol{b}}^{T} \boldsymbol{B}_{\boldsymbol{b}} \\
-\hat{B} \boldsymbol{B}_{\boldsymbol{b}}^{T} \boldsymbol{B}_{\boldsymbol{u}}-\hat{B} \boldsymbol{B}_{\boldsymbol{u}}^{T} \boldsymbol{B}_{\boldsymbol{b}}
\end{array}\right) d x \\
& \boldsymbol{N}_{1}=b \int_{0}^{l_{e}}\left(\begin{array}{c}
\hat{A} \boldsymbol{B}_{u}^{T} \boldsymbol{B}_{N L}+\hat{A} \boldsymbol{B}_{N L}^{T} \boldsymbol{B}_{u} \\
-\hat{B} \boldsymbol{B}_{b}^{T} \boldsymbol{B}_{N L}-\hat{B} \boldsymbol{B}_{N L}^{T} \boldsymbol{B}_{b} \\
+\hat{A} \boldsymbol{B}_{u} u \tilde{\boldsymbol{G}}^{T} \tilde{\boldsymbol{G}}-\hat{B} \boldsymbol{B}_{b} u \tilde{\boldsymbol{G}}^{T} \tilde{\boldsymbol{G}}
\end{array}\right) d x \\
& \boldsymbol{N}_{2}=b \int_{0}^{l e}\left(\hat{A} \boldsymbol{B}_{N L}^{T} \boldsymbol{B}_{N L}+\hat{A}\left(\frac{1}{2} \boldsymbol{B}_{N L} \boldsymbol{u}\right) \tilde{\boldsymbol{G}}^{T} \tilde{\boldsymbol{G}}\right) d x
\end{aligned}
$$

$\tilde{\boldsymbol{G}}$ and $\boldsymbol{B}_{N L}$ can be derived by considering $\theta=\tilde{\boldsymbol{G}} \boldsymbol{u}, \boldsymbol{B}_{N L}=\tilde{A} \tilde{\boldsymbol{G}}$ and Eq. (13). The stretching $(\hat{A})$, bending $(\hat{D})$ and stretchingbending coupling $(\hat{B})$ stiffnesses are determined [28] as

$$
(\hat{A}, \hat{B}, \hat{D})=\int_{-\frac{h}{2}}^{\frac{h}{2}}\left(1, z, z^{2}\right) E(z) d z
$$

The material variation in $z$ direction leads to the change of the neutral axis position. $h_{0}$ is the distance between the neutral axis and the centroid of the cross-section area as shown in Fig. 2.

$$
h_{0}=\frac{\hat{B} \bar{E}-\hat{D} \kappa}{\hat{A} \bar{E}-\hat{B} \kappa}
$$

where $\bar{E}=\left(\boldsymbol{B}_{u}+\frac{1}{2} \boldsymbol{B}_{N L}\right) \boldsymbol{u}$ and $\kappa=\frac{\partial^{2} w}{\partial x^{2}}$. The distance $h_{0}$ can be simplified in the start of loading and before buckling as $h_{0}=\frac{\hat{B}}{A}$ in which the curvature, $\kappa$, is zero. Accordingly, compressive axial load produces a moment in the end

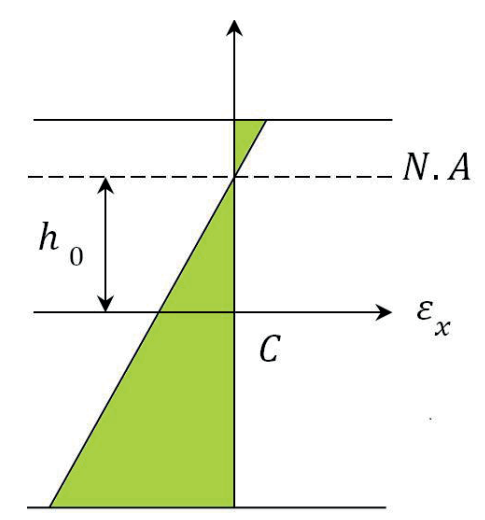

Fig. 2 Variation of strain through thickness supports of FGM columns due to stretching-bending coupling stiffness. Moreover, increasing axial load causes that the neutral axis changes with respect to the curvature.

\subsubsection{Mechanical loading}

The variation of the total potential energy in the mechanical loading can be obtained as follows

$$
\delta \Pi=\delta \boldsymbol{u}^{T} \boldsymbol{R}-\delta \boldsymbol{u}^{T} \eta \boldsymbol{F}_{\text {ext }},
$$

in which $\boldsymbol{R}, \boldsymbol{F}_{\text {ext }}$ and $\eta$ are the internal force, the external load vector and load level, respectively. It can be rewritten for the Euler-Bernoulli beam discretized by $n_{e}$ elements as

$$
\delta \Pi=\bigcup_{e=1}^{n_{e}} b \int_{0}^{l e} \int_{-\frac{h}{2}}^{\frac{h}{2}} \delta \varepsilon_{x} \sigma_{x} d z d x-\delta \boldsymbol{u}^{T} \eta \boldsymbol{F}_{\text {ext }} .
$$

The operator, $\cup$, denotes an assembly process.

Accordingly, the internal force, $\boldsymbol{R}$, is derived from the first term on the right side of Eq. (21) as

$$
\boldsymbol{R}=\bigcup_{e=1}^{n_{e}} b \int_{0}^{l e} \int_{-\frac{h}{2}}^{\frac{h}{2}}\left(\boldsymbol{B}_{0}+\boldsymbol{B}_{N L}\right)^{T} \sigma_{x} d z d x
$$

The Taylor series expansion is used to solve the nonlinear equilibrium equation using the iterative NewtonRaphson algorithm as

$$
\delta \Pi(\overline{\boldsymbol{u}}+\Delta \boldsymbol{u}, \bar{\eta})=\delta \Pi(\overline{\boldsymbol{u}}, \bar{\eta})+D \delta \Pi(\overline{\boldsymbol{u}}, \bar{\eta}) \cdot \Delta \boldsymbol{u}+\operatorname{Re},
$$

where $\overline{\boldsymbol{u}}$ is an already known displacement vector and $R e$ is the residuum of the Taylor series which is neglected to obtain the linear equation system based on an iterative solution. $D \delta \Pi$ denotes the gradient vector of $\delta \Pi$ at $\overline{\boldsymbol{u}}$. Also, $\Delta \delta \Pi$ is used to simplify the notation of the directional derivative in the Taylor series expansion as

$\Delta \delta \Pi=D \delta \Pi(\overline{\boldsymbol{u}}, \bar{\eta}) \cdot \Delta \boldsymbol{u}$.

Therefore, the linearization of the virtual work yields

$$
\Delta \delta \Pi=-\delta \Pi .
$$

By considering

$$
\Delta \delta \Pi=\delta \boldsymbol{u}^{T} \bigcup_{e=1}^{n_{e}} \boldsymbol{K}_{T} \Delta \boldsymbol{u},
$$

and by inserting Eqs. (20) and (26) into Eq. (25), the equilibrium equation system is derived as

$$
\bigcup_{e=1}^{n_{e}} \boldsymbol{K}_{T} \Delta \boldsymbol{u}=-\left(\boldsymbol{R}-\bar{\eta} \boldsymbol{F}_{\text {ext }}\right) .
$$

The displacement increments can be computed by the load control algorithm in the given load level $\bar{\eta}$. 


\subsubsection{Thermal loading}

A literature review on the thermal post-buckling subject using the FEM shows that the solution of the nonlinear thermal equilibrium equation is performed through two techniques: A) employing a direct iterative procedure without the linearization of the equation and without the determination of the tangent stiffness matrix [31], B) the linearization of the equilibrium equation and the determination of the tangent stiffness matrix [32]. Relations and results in this research work have been represented based on the second one. In both techniques, the combined Newton-Raphson/Arclength procedure has been applied to solve the nonlinear equation. The arc-length will generally be advantageous where bifurcation or limit points occur [33]. In the load control and the displacement control, the load level or the displacement level are kept constant and iterations are performed until a state of equilibrium is reached [34]. The total potential energy by considering thermal loading is obtained as [35]

$\Pi=\bigcup_{e=1}^{n_{e}} b \int_{0}^{l e} \int_{-\frac{h}{2}}^{\frac{h}{2}}\left(\frac{1}{2} \varepsilon_{x} \sigma_{x}-\varepsilon_{x} \sigma_{t h}\right) d z d x$,

where the thermal stress can be found as

$\sigma_{t h}=E(z) \alpha(z) \Delta_{0} T$.

The variation of the total potential energy is

$$
\delta \Pi=\delta \boldsymbol{u}^{T} \boldsymbol{R}-\delta \boldsymbol{u}^{T} \Delta_{0} T \overline{\boldsymbol{F}}_{t h},
$$

in which the thermal force vector is obtained by inserting Eq. (29) into Eq. (28) as

$$
\overline{\boldsymbol{F}}_{t h}=\bigcup_{e=1}^{n_{e}} b \int_{0}^{l e} \int_{-\frac{h}{2}}^{\frac{h}{2}}\left(\boldsymbol{B}_{0}+\boldsymbol{B}_{N L}\right)^{T} E(z) \alpha(z) d z d x
$$

Since the thermal force vector, $\overline{\boldsymbol{F}}_{t h}$, is including both displacement and load terms, the load control fail to solve such equilibrium equation. If the term including the displacement in the thermal force vector $\left(\boldsymbol{B}_{N L}\right)$ is neglected, the load control can be successfully used. By considering the nonlinear term of $\boldsymbol{B}_{N L}$, the linearization of the variation of the potential energy is performed as follows

$$
\begin{aligned}
& \delta \Pi\left(\overline{\boldsymbol{u}}+\Delta \boldsymbol{u}, \Delta_{0} T+\Delta\left(\Delta_{0} T\right)\right)=\delta \Pi\left(\overline{\boldsymbol{u}}, \Delta_{0} T\right) \\
& +D \delta \Pi\left(\overline{\boldsymbol{u}}, \Delta_{0} T\right) \cdot \Delta \boldsymbol{u}+D \delta \Pi\left(\overline{\boldsymbol{u}}, \Delta_{0} T\right) \cdot \Delta\left(\Delta_{0} T\right)+R e
\end{aligned}
$$

Considering the equilibrium state, an incremental iterative solution is proposed to determine the updated displacement corresponding to thermal load as
$D \delta \Pi\left(\overline{\boldsymbol{u}}, \Delta_{0} T\right) \cdot \Delta \boldsymbol{u}+D \delta \Pi\left(\overline{\boldsymbol{u}}, \Delta_{0} T\right) \cdot \Delta\left(\Delta_{0} T\right)$

$=-\delta \Pi\left(\overline{\boldsymbol{u}}, \Delta_{0} T\right)$,

in which the first term can be explained as

$$
D \delta \Pi\left(\overline{\boldsymbol{u}}, \Delta_{0} T\right) \cdot \Delta \boldsymbol{u}=\delta \boldsymbol{u}^{T} \bigcup_{e=1}^{n_{e}} \boldsymbol{K}_{T} \Delta \boldsymbol{u}
$$

The tangent stiffness matrix is obtained as

$\boldsymbol{K}_{T}=\boldsymbol{K}_{0}+\boldsymbol{N}_{1}+\boldsymbol{N}_{2}-\boldsymbol{K}_{t h}$,

where

$\boldsymbol{K}_{t h}=b \int_{0}^{l e} \int_{-\frac{h}{2}}^{\frac{h}{2}} \tilde{\boldsymbol{G}}^{T} \sigma_{t h} \tilde{\boldsymbol{G}} d z d x$.

The second term of Eq. (33) can be recast with

$D \delta \Pi\left(\overline{\boldsymbol{u}}, \Delta_{0} T\right) . \Delta\left(\Delta_{0} T\right)=-\delta \boldsymbol{u}^{T} \overline{\boldsymbol{F}}_{t h} \Delta\left(\Delta_{0} T\right)$.

Substituting Eqs. (30), (34) and (37) into Eq. (33) gives

$\bigcup_{e=1}^{n_{e}} \boldsymbol{K}_{T} \Delta \boldsymbol{u}-\overline{\boldsymbol{F}}_{t h} \Delta\left(\Delta_{0} T\right)=-\left(\boldsymbol{R}-\overline{\boldsymbol{F}}_{t h} \Delta_{0} T\right)$.

The matrix form to use the arc-length algorithm is presented as

$\left[\begin{array}{cc}\bigcup_{e=1}^{n_{e}} \boldsymbol{K}_{T} & -\overline{\boldsymbol{F}}_{t h} \\ \boldsymbol{f}^{T} & f_{, \eta}\end{array}\right]\left\{\begin{array}{c}\Delta \boldsymbol{u} \\ \Delta\left(\Delta_{0} T\right)\end{array}\right\}=-\left\{\begin{array}{c}\left.\boldsymbol{R}-\overline{\boldsymbol{F}}_{t h} \Delta_{0} T\right\} \\ f\end{array}\right\}$,

where the constraint equation function and its gradient (i.e. $f$ and $\boldsymbol{f}^{T}$, respectively) can be found in [34]. Two temperature distributions of uniform and linear tabulated in Table 1 are considered to perform the thermal buckling analysis.

\subsection{Incorporating crack in the beam element}

The most significant part of the finite element analysis of a beam including the crack is related to cracked elements. A cracked element in the beam is divided into three sub-elements as shown in Fig. 3

These sub-elements are connected to each other using the following five continuity conditions at $x=x_{0}$ as follows

$u_{e L}=u_{e R} \rightarrow u_{2}=u_{3}$,

$w_{e L}=w_{e R} \rightarrow w_{2}=w_{3}$,

Table 1 Temperature distribution

\begin{tabular}{lc}
\hline Type of thermal loading & Temperature (T) \\
\hline Uniform & $T=T_{c}=T_{m}$ \\
Linear & $T=T_{m}+\left(T_{c}-T_{m}\right)\left(\frac{1}{2}+\frac{z}{h}\right)$ \\
\hline
\end{tabular}




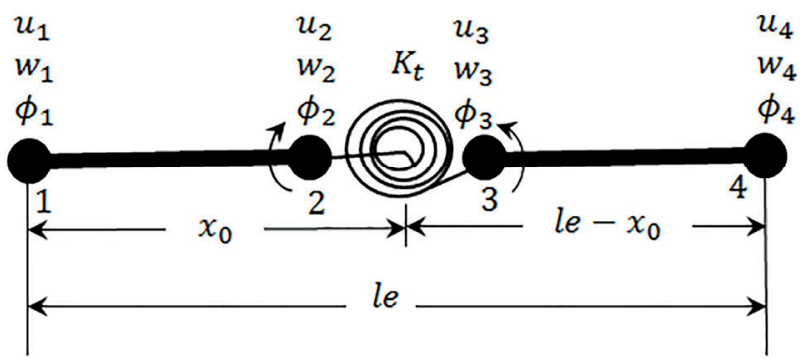

Fig. 3 Cracked element

$$
\begin{aligned}
& w_{e L}^{\prime}+\frac{(\hat{B} \bar{E} b-\hat{D} \kappa b)}{K_{t}}=w_{e R}^{\prime} \\
& M_{e L}=M_{e R} \rightarrow(\hat{B} \bar{E} b-\hat{D} \kappa b)_{e L}=(\hat{B} \bar{E} b-\hat{D} \kappa b)_{e R} \\
& V_{e L}=V_{e R} \rightarrow\left(\hat{B} \frac{\partial \bar{E}}{\partial x} b-\hat{D} \frac{\partial \kappa}{\partial x} b\right)_{e L}=\left(\hat{B} \frac{\partial \bar{E}}{\partial x} b-\hat{D} \frac{\partial \kappa}{\partial x} b\right)_{e R}
\end{aligned}
$$

The expanded form of Eqs. (42) to (44) can be found in "Appendix A". The stiffness matrices for a cracked element are enriched by considering crack characteristics and continuity conditions. Two techniques of LM and CM are introduced to insert crack parameters into the stiffness matrices. The substantial difference between the two techniques is highlighted when the influence of the material inhomogeneity is studied in a structure like a beam made of FGM.

\subsection{Lagrange Multiplier technique}

The LM technique is applied to derive the finite element parameters of the cracked element. This technique is effective and useful in solving nonlinear problems where inserting geometrical non-linearity relations and material inhomogeneity into continuity conditions yields a set of complicated equations. Since continuity conditions in the present research have a nonlinear nature, using the $\mathrm{CM}$ technique instead of the LM one results in error in the analysis. In other words, the CM technique is implemented by ignoring the geometrically nonlinear term, $\frac{1}{2}\left(\frac{\partial w}{\partial x}\right)^{2}$, in continuity conditions. While in the LM technique, the five continuity conditions are added to the total potential energy as fully constrained conditions without any simplification. A plan is considered to derive the enriched stiffness matrices in which the main equation is the total potential energy. The crack parameters are involved through inserting continuity conditions into the potential energy as follows

$$
\begin{aligned}
\Pi_{\text {crack }} & =U_{L}+U_{R}+U_{\text {spring }}+\Omega_{\text {crack }} \\
& +\lambda_{1} H_{1}+\lambda_{2} H_{2}+\lambda_{3} H_{3}+\lambda_{4} H_{4}+\lambda_{5} H_{5}
\end{aligned}
$$

in which $U_{L}, U_{R}$ and $U_{\text {spring }}$ are strain energies of left and right-hand-side sub-elements and the rotational spring, respectively, and the potential energy of external forces is denoted by $\Omega_{\text {crack }}$. Also, Lagrange multipliers, $\lambda_{1}$ to $\lambda_{5}$, are related to five continuity equations. $H_{1}$ to $H_{2}$ are defined as the constraints of continuity in a cracked element, which are obtained according to Eqs. (40) to (44).

$$
\begin{aligned}
H_{1} & =u_{2}-u_{3} \\
H_{2} & =w_{2}-w_{3} \\
H_{3} & =a_{3} w_{2}+b_{3} \phi_{2}^{2}+c_{3} \phi_{2}-\phi_{3}+d_{3} u_{1}+e_{3} w_{1}+f_{3} \phi_{1}+g_{3} u_{4} \\
H_{4} & =a_{4} w_{2}+b_{4} \phi_{2}^{2}+c_{4} \phi_{2}+d_{4} \phi_{3}+e_{4} \phi_{3}^{2} \\
& +f_{4} w_{1}+g_{4} \phi_{1}+h_{4} w_{4}+r_{4} \phi_{4} \\
H_{5} & =a_{5} w_{1} \phi_{2}+b_{5} \phi_{1} \phi_{2}+c_{5} w_{2} \phi_{2}+d_{5} \phi_{2}^{2} \\
& +e_{5} w_{1}+f_{5} \phi_{1}+g_{5} w_{2}+h_{5} \phi_{2}+k_{5} w_{2} \phi_{3}+l_{5} \phi_{3}^{2} \\
& +m_{5} w_{4} \phi_{3}+n_{5} \phi_{3} \phi_{4}+s_{5} w_{2}+y_{5} \phi_{3}+r_{5} w_{4}+t_{5} \phi_{4}
\end{aligned}
$$

Coefficients of Eqs. (46) to (50) are determined by inserting the shape functions into Eqs. (40) to (44) as follows

$a_{3}=\frac{6 \hat{D} b}{K_{t} x_{0}{ }^{2}}, b_{3}=-\frac{\hat{B} b}{2 K_{t}}, c_{3}=-\frac{4 \hat{D} b}{K_{t} x_{0}}, d_{3}=-\frac{\hat{B} b}{K_{t} l e}$,

$e_{3}=-\frac{6 \hat{D} b}{K_{t} x_{0}{ }^{2}}, f_{3}=-\frac{2 \hat{D} b}{K_{t} x_{0}}, g_{3}=\frac{\hat{B} b}{K_{t} l e}$,

$a_{4}=\left(\frac{6 \hat{D} b}{x_{0}{ }^{2}}-\frac{6 \hat{D} b}{\left(l e-x_{0}\right)^{2}}\right), b_{4}=\frac{\hat{B} b}{2}, c_{4}=-\frac{4 \hat{D} b}{x_{0}}, d_{4}=-\frac{4 \hat{D} b}{\left(l e-x_{0}\right)}$,

$e_{4}=-\frac{\hat{B} b}{2}, f_{4}=-\frac{6 \hat{D} b}{x_{0}{ }^{2}}, g_{4}=-\frac{2 \hat{D} b}{x_{0}}, h_{4}=\frac{6 \hat{D} b}{\left(l e-x_{0}\right)^{2}}$,

$r_{4}=-\frac{2 \hat{D} b}{\left(l e-x_{0}\right)}, a_{5}=\frac{6 \hat{B} b}{x_{0}{ }^{2}}, b_{5}=\frac{2 \hat{B} b}{x_{0}}, c_{5}=-\frac{6 \hat{B} b}{x_{0}{ }^{2}}$,

$d_{5}=\frac{4 \hat{B} b}{x_{0}}, e_{5}=-\frac{12 \hat{D} b}{x_{0}{ }^{3}}, f_{5}=-\frac{6 \hat{D} b}{x_{0}{ }^{2}}, g_{5}=\frac{12 \hat{D} b}{x_{0}{ }^{3}}$,

$h_{5}=-\frac{6 \hat{D} b}{x_{0}{ }^{2}}, k_{5}=\frac{6 \hat{B} b}{\left(l e-x_{0}\right)^{2}}, l_{5}=\frac{4 \hat{B} b}{\left(l e-x_{0}\right)}, m_{5}=-\frac{6 \hat{B} b}{\left(l e-x_{0}\right)^{2}}$,

$n_{5}=\frac{2 \hat{B} b}{\left(l e-x_{0}\right)}, s_{5}=\frac{12 \hat{D} b}{\left(l e-x_{0}\right)^{3}}, y_{5}=\frac{6 \hat{D} b}{\left(l e-x_{0}\right)^{2}}, r_{5}=-\frac{12 \hat{D} b}{\left(l e-x_{0}\right)^{3}}$,

$t_{5}=\frac{6 \hat{D} b}{\left(l e-x_{0}\right)^{2}}$

The variation of Eq. (45) yields the equilibrium equation of the cracked element whose secant stiffness matrix can be specified as 


$$
\begin{aligned}
& \delta \Pi_{\text {crack }}=\delta U_{L}+\delta U_{R}+\delta U_{\text {spring }}+\delta \Omega_{\text {crack }}+\delta\left(\lambda_{1} H_{1}\right) \\
& +\delta\left(\lambda_{2} H_{2}\right)+\delta\left(\lambda_{3} H_{3}\right)+\delta\left(\lambda_{4} H_{4}\right)+\delta\left(\lambda_{5} H_{5}\right)=0 \rightarrow \boldsymbol{K}_{\text {S.crack }}
\end{aligned}
$$

The linearization of the equilibrium equation is performed by using the increment of Eq. (52) as follows:

$$
\begin{aligned}
& \Delta \delta \Pi_{\text {crack }}=\Delta \delta U_{L}+\Delta \delta U_{R}+\Delta \delta U_{\text {spring }}+\Delta \delta \Omega_{\text {crack }} \\
& +\Delta \delta\left(\lambda_{1} H_{1}\right)+\Delta \delta\left(\lambda_{2} H_{2}\right)+\Delta \delta\left(\lambda_{3} H_{3}\right) \\
& +\Delta \delta\left(\lambda_{4} H_{4}\right)+\Delta \delta\left(\lambda_{5} H_{5}\right)=-\delta \Pi_{\text {crack }} \rightarrow \boldsymbol{K}_{\text {T.crack }},
\end{aligned}
$$

which gives the tangent stiffness matrix of the cracked element.

In order to obtain the tangent stiffness matrix of the cracked element in non-linear finite element analysis. The procedure represented in Eqs. (54) to (60) is applied to recast the ith term of Eq. (53) in the form of $\delta \boldsymbol{u}^{T} \boldsymbol{K}_{T i}(\boldsymbol{u}) \Delta \boldsymbol{u}$.

$$
\begin{aligned}
& \Delta \delta U_{L}=\delta \boldsymbol{u}^{T} \boldsymbol{K}_{T L}(\boldsymbol{u}) \Delta \boldsymbol{u} \\
& \Delta \delta U_{R}=\delta \boldsymbol{u}^{T} \boldsymbol{K}_{T R}(\boldsymbol{u}) \Delta \boldsymbol{u} \\
& \Delta \delta\left(\lambda_{1} H_{1}\right)=\delta \lambda_{1}\left(\Delta u_{2}-\Delta u_{3}\right)+\Delta \lambda_{1} \delta\left(u_{2}-u_{3}\right) \\
& \Delta \delta\left(\lambda_{2} H_{2}\right)=\delta \lambda_{2}\left(\Delta w_{2}-\Delta w_{3}\right)+\Delta \lambda_{2} \delta\left(w_{2}-w_{3}\right) \\
& \Delta \delta\left(\lambda_{3} H_{3}\right)=\delta \lambda_{3}\left(\begin{array}{l}
a_{3} \Delta w_{2}+2 b_{3} \phi_{2} \Delta \phi_{2}+c_{3} \Delta \phi_{2}-\Delta \phi_{3} \\
+d_{3} \Delta u_{1}+e_{3} \Delta w_{1}+f_{3} \Delta \phi_{1}+g_{3} \Delta u_{4}
\end{array}\right) \\
& +\Delta \lambda_{3}\left(\begin{array}{l}
a_{3} \delta w_{2}+2 b_{3} \phi_{2} \delta \phi_{2}+c_{3} \delta \phi_{2}-\delta \phi_{3} \\
+d_{3} \delta u_{1}+e_{3} \delta w_{1}+f_{3} \delta \phi_{1}+g_{3} \delta u_{4}
\end{array}\right) \\
& +2 \lambda_{3} b_{3} \Delta \phi_{2} \delta \phi_{2}
\end{aligned}
$$

$$
\begin{aligned}
\Delta \delta\left(\lambda_{4} H_{4}\right) & =\delta \lambda_{4}\left(\begin{array}{l}
a_{4} \Delta w_{2}+2 b_{4} \phi_{2} \Delta \phi_{2}+c_{4} \Delta \phi_{2}+d_{4} \Delta \phi_{3} \\
+2 e_{4} \phi_{3} \Delta \phi_{3}+f_{4} \Delta w_{1}+g_{4} \Delta \phi_{1}+h_{4} \Delta w_{4}+r_{4} \Delta \phi_{4}
\end{array}\right) \\
& \left.+\Delta \lambda_{4}\left(\begin{array}{l}
a_{4} \delta w_{2}+2 b_{4} \phi_{2} \delta \phi_{2}+c_{4} \delta \phi_{2}+d_{4} \delta \phi_{3} \\
+2 e_{4} \phi_{3} \delta \phi_{3}+f_{4} \delta w_{1}+g_{4} \delta \phi_{1}+h_{4} \delta w_{4}+r_{4} \delta \phi_{4}
\end{array}\right)\right) \\
& +\lambda_{4}\left(2 b_{4} \Delta \phi_{2} \delta \phi_{2}+2 e_{4} \Delta \phi_{3} \delta \phi_{3}\right)
\end{aligned}
$$

The tangent stiffness matrix of the cracked element is determined by the sum of matrices obtained from "Appendix B".

$$
\boldsymbol{K}_{\text {T.crack }}=\boldsymbol{K}_{T L}+\boldsymbol{K}_{T R}+\boldsymbol{K}_{\text {spring }}+\boldsymbol{K}_{T 1}+\ldots+\boldsymbol{K}_{T 5}
$$

\subsection{Conversion Matrix technique}

The CM technique is implemented within the finite element framework based on a conversion in which the displacements of the middle nodes are written in terms of the displacements of basic nodes. A simplification in CM technique is considered to derive relations. Accordingly, the nonlinear part of the kinematic equation in the continuity conditions is neglected. A basic characteristic of the CM is related to the order of degrees of freedom for the cracked element, as the order is without changing in the CM, unlike the LM. In other words, cracked elements in the CM and the LM have 6 and 17 degrees of freedom, respectively. Two conversion matrices, $\boldsymbol{C}_{L}$ and $\boldsymbol{C}_{R}$, are introduced to derive the enriched stiffness matrices. The cracked element is divided into three parts including two sub-elements and a rotational spring. The strain energies corresponding to each part are determined in terms of displacements of the basic nodes (first and fourth) as shown in Fig. 3. If the nonlinear terms of the continuity conditions are ignored, a linear relation is established between displacements of the middle nodes (second and third nodes) and the basic nodes. Therefore, in the CM, Eqs. (42) to (44) are simplified by neglecting the nonlinear part as follows

$$
\begin{aligned}
& \frac{6 c_{1} b}{x_{0}{ }^{2}} w_{2}-\left(1+\frac{4 c_{1} b}{x_{0}}\right) \phi_{2}+\phi_{3}=\frac{c_{1} b}{x_{0}{ }^{2}} w_{1}+\frac{2 c_{1} b}{x_{0}} \phi_{1}, \\
& 6 c_{1} b\left(\frac{1}{x_{0}{ }^{2}}-\frac{1}{\left(l e-x_{0}\right)^{2}}\right) w_{2}-\frac{4 c_{1} b}{x_{0}} \phi_{2}-\frac{4 c_{1} b}{\left(l e-x_{0}\right)} \phi_{3} \\
& =\frac{6 c_{1} b}{x_{0}{ }^{2}} w_{1}+\frac{2 c_{1} b}{x_{0}} \phi_{1}-\frac{6 c_{1} b}{\left(l e-x_{0}\right)^{2}} w_{4}-\frac{2 c_{1} b}{\left(l e-x_{0}\right)} \phi_{4}
\end{aligned}
$$

$+\lambda_{5}\left(\begin{array}{l}a_{5} \delta w_{1} \Delta \phi_{2}+a_{5} \Delta w_{1} \delta \phi_{2}+b_{5} \delta \phi_{1} \Delta \phi_{2}+b_{5} \Delta \phi_{1} \delta \phi_{2}+c_{5} \delta w_{2} \Delta \phi_{2}+c_{5} \Delta w_{2} \delta \phi_{2}+2 d_{5} \Delta \phi_{2} \delta \phi_{2} \\ +k_{5} \delta w_{2} \Delta \phi_{3}+k_{5} \Delta w_{2} \delta \phi_{3}+2 l_{5} \Delta \phi_{3} \delta \phi_{3}+m_{5} \delta w_{4} \Delta \phi_{3}+m_{5} \Delta w_{4} \delta \phi_{3}+n_{5} \Delta \phi_{4} \delta \phi_{3}+n_{5} \delta \phi_{4} \Delta \phi_{3}\end{array}\right)$

$+\Delta \lambda_{5}\left(\begin{array}{l}a_{5} \delta w_{1} \phi_{2}+a_{5} w_{1} \delta \phi_{2}+b_{5} \delta \phi_{1} \phi_{2}+b_{5} \phi_{1} \delta \phi_{2}+c_{5} \delta w_{2} \Delta \phi_{2}+c_{5} w_{2} \delta \phi_{2}+2 d_{5} \phi_{2} \delta \phi_{2}+e_{5} \delta w_{1}+f_{5} \delta \phi_{1}+g_{5} \delta w_{2}+h_{5} \delta \phi_{2} \\ +k_{5} \delta w_{2} \phi_{3}+k_{5} w_{2} \delta \phi_{3}+2 l_{5} \phi_{3} \delta \phi_{3}+m_{5} \delta w_{4} \phi_{3}+m_{5} w_{4} \delta \phi_{3}+n_{5} \phi_{4} \delta \phi_{3}+n_{5} \delta \phi_{4} \phi_{3} s_{5} \delta w_{2}+y_{5} \delta \phi_{3}+r_{5} \delta w_{4}+t_{5} \delta \phi_{4}\end{array}\right)$ 


$$
\begin{aligned}
& 12 c_{1} b\left(\frac{1}{x_{0}{ }^{3}}+\frac{1}{\left(l e-x_{0}\right)^{3}}\right) w_{2}-\frac{6 c_{1} b}{x_{0}{ }^{2}} \phi_{2}+\frac{6 c_{1} b}{\left(l e-x_{0}\right)^{2}} \phi_{3} \\
& =\frac{12 c_{1} b}{x_{0}{ }^{3}} w_{1}+\frac{6 c_{1} b}{x_{0}{ }^{2}} \phi_{1}+\frac{12 c_{1} b}{\left(l e-x_{0}\right)^{3}} w_{4}-\frac{6 c_{1} b}{\left(l e-x_{0}\right)^{2}} \phi_{4},
\end{aligned}
$$

in which

$$
\left.c_{1}=\frac{0.25 h^{3}}{\ln \left(\frac{E_{1}}{E_{2}}\right)^{3} K_{t}\left(+E_{1} \ln \left(\frac{E_{1}}{E_{2}}\right)^{2}-4 \ln \left(\frac{E_{1}}{E_{2}}\right)\right.}\right)
$$

The strain energy of the cracked element is considered as the sum of energies of the three parts.

$U_{\text {crack }}=U_{L}+U_{R}+U_{\text {spring }}$

The increment of strain energy variation is

$$
\Delta \delta U_{\text {crack }}=\Delta \delta U_{L}+\Delta \delta U_{R}+\Delta \delta U_{\text {spring }} .
$$

The components of $\Delta \delta U_{\text {crack }}$ can be written as follows

$$
\begin{aligned}
& \Delta \delta U_{L}=\delta \boldsymbol{u}_{L}{ }^{T} \boldsymbol{k}_{T L} \Delta \boldsymbol{u}_{L}, \Delta \delta U_{R}=\delta \boldsymbol{u}_{R}{ }^{T} \boldsymbol{k}_{T R} \Delta \boldsymbol{u}_{R}, \\
& \Delta \delta U_{\text {spring }}=\delta \phi^{T} K_{t} \Delta \phi .
\end{aligned}
$$

The conversion matrices can be derived as

$$
\boldsymbol{u}_{L}=\boldsymbol{C}_{L} \boldsymbol{u}, \boldsymbol{u}_{R}=\boldsymbol{C}_{R} \boldsymbol{u}, \phi=\boldsymbol{C}_{\text {spring }} \boldsymbol{u} .
$$

Inserting Eqs. (69) and (68) into Eq. (67) yields

$$
\begin{aligned}
& \Delta \delta U_{\text {crack }}=\delta \boldsymbol{u}^{T} \boldsymbol{C}_{L}{ }^{T} \boldsymbol{k}_{T L}(\boldsymbol{u}) \boldsymbol{C}_{L} \Delta \boldsymbol{u}+ \\
& \delta \boldsymbol{u}^{T} \boldsymbol{C}_{R}{ }^{T} \boldsymbol{k}_{T R}(\boldsymbol{u}) \boldsymbol{C}_{R} \Delta \boldsymbol{u}+\delta \boldsymbol{u}^{T} \boldsymbol{C}_{\text {spring }}{ }^{T} K_{t} \boldsymbol{C}_{\text {spring }} \Delta \boldsymbol{u} .
\end{aligned}
$$

Therefore, the tangent stiffness matrix for the cracked element can be extracted as follows

$$
\boldsymbol{K}_{\text {T.crack }}=\boldsymbol{K}_{T L}+\boldsymbol{K}_{T R}+\boldsymbol{K}_{\text {spring }},
$$

in which

$$
\begin{aligned}
& \boldsymbol{K}_{T L}=\boldsymbol{C}_{L}^{T} \boldsymbol{k}_{T L} \boldsymbol{C}_{L}, \\
& \boldsymbol{K}_{T R}=\boldsymbol{C}_{R}{ }^{T} \boldsymbol{k}_{T R} \boldsymbol{C}_{R}, \\
& \boldsymbol{K}_{\text {spring }}=\boldsymbol{C}_{\text {spring }}{ }^{T} K_{t} \boldsymbol{C}_{\text {spring }},
\end{aligned}
$$

and $\boldsymbol{C}_{L}, \boldsymbol{C}_{R}$ and $\boldsymbol{C}_{\text {Spring }}$ can be found in [25].

\section{Results and discussion}

Table 2 shows the material properties of the metal-ceramic FGM beam used as a case study in mechanical and thermal post-buckling analysis, in which length, height, and width are considered as $L=2 \mathrm{~m}, h=0.1 \mathrm{~m}$ and $b=0.1 \mathrm{~m}$, respectively. Two sets of boundary conditions including clampedclamped(C-C) and simply supported-simply supported (SS-SS) are considered. In thermal loading, two types of uniform and linear temperature distribution are assumed.

Table 3 compares the critical buckling load values for the intact FGM column calculated using the analytical and FEM. An eigen-value solution is performed in the FE analysis.

The convergence of the linear buckling analysis, as shown in Fig. 4(a), explains that desired results are even obtained with 10 elements. In other words, the difference almost $0.5 \%$ is observed between the minimum and maximum critical buckling loads. Fig. 4(b) demonstrates that after about 51 elements, the change in critical buckling magnitude is negligible, so this element number is adopted for other analyses in this paper.

In Fig. 4(b), the convergence of FEM results in the non-linear buckling analysis is investigated for the C-C FGM beam containing crack incorporated in the middle of the beam with $a / h=0.5$.

Table 2 Material properties of FGM beam

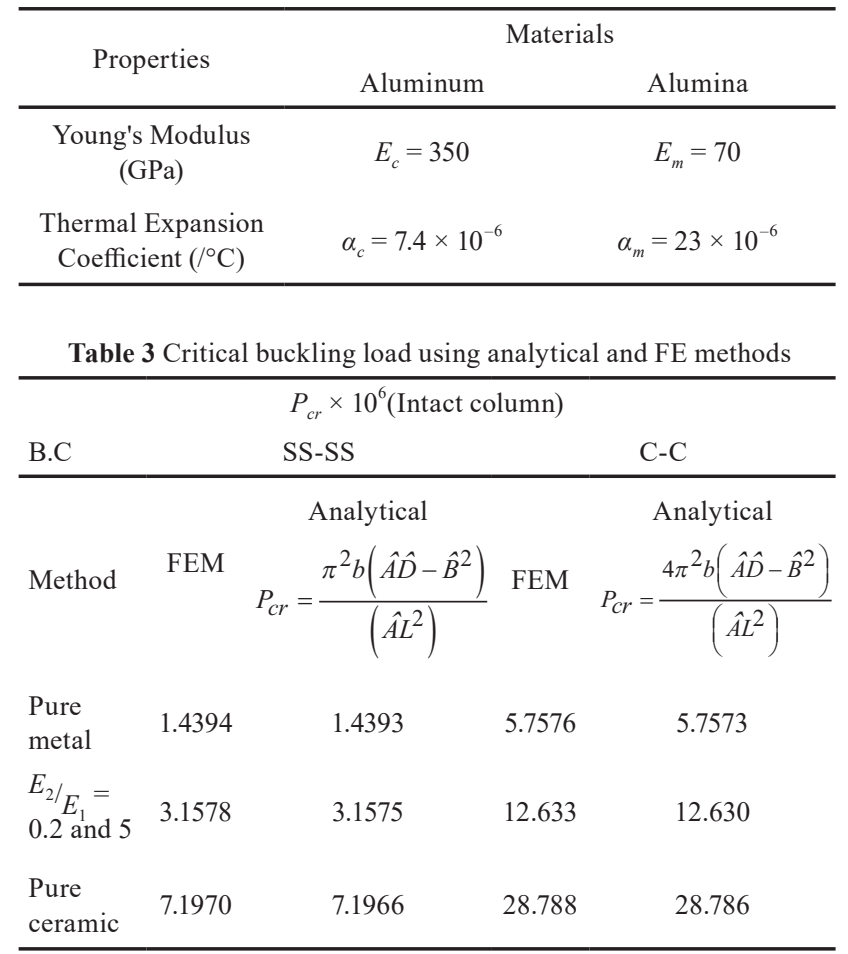




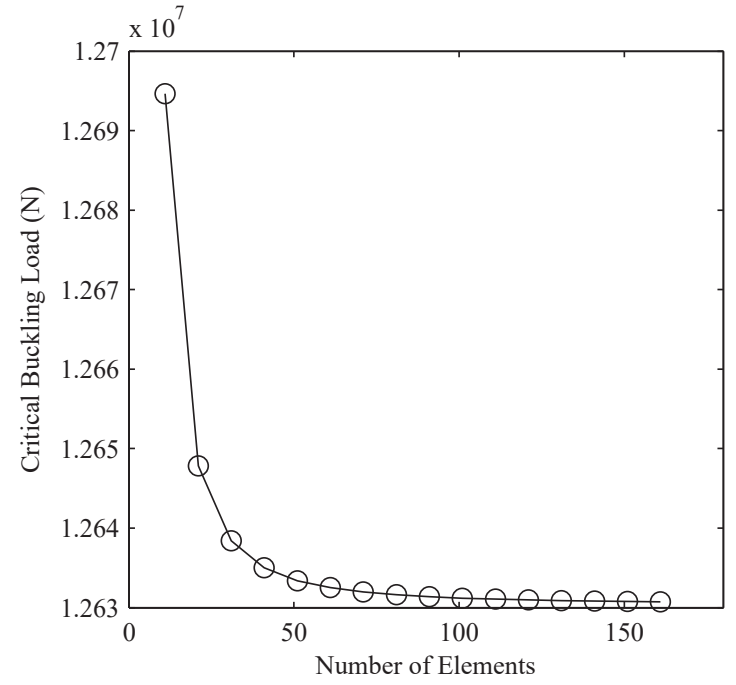

(a)

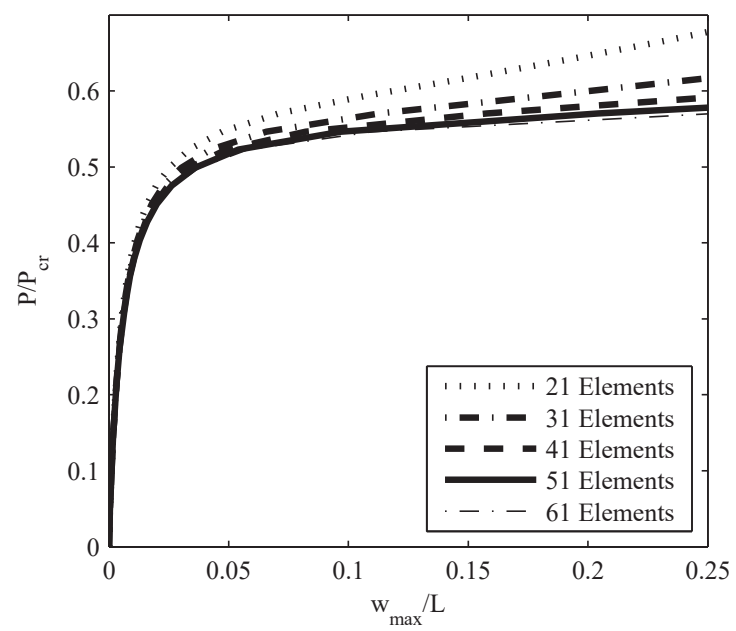

(b)

Fig. 4 Convergence behavior of FE analysis for C-C FGM beam and

${ }^{E_{2}} E_{1}=0.2$ : a) Linear Buckling of Intact beam, b) Post-buckling of Cracked beam with ${ }^{a / h}=0.5,{ }^{X_{0}} L=0.5$

Fig. 5 displays the comparison of two CM and LM techniques in the post-buckling analysis of the FGM cracked column. Fig. 5(a) shows the pre- and post-buckling behavior of the column under uniform thermal load in two crack depths in which close accordance between two techniques is observed. On the other hand in mechanical loading shown in Fig. 5(b), a clear difference is recognized due to neglecting the non-linear part in the continuity conditions. Indeed, applying the non-linear term in continuity conditions of the LM technique leads to higher level of stiffness of the structure which can be obviously seen in Fig. 5(b). The representation of a straight-forward fact about the difference and the closeness of the two techniques is difficult due to the simultaneous effects and interaction of some parameters listed as

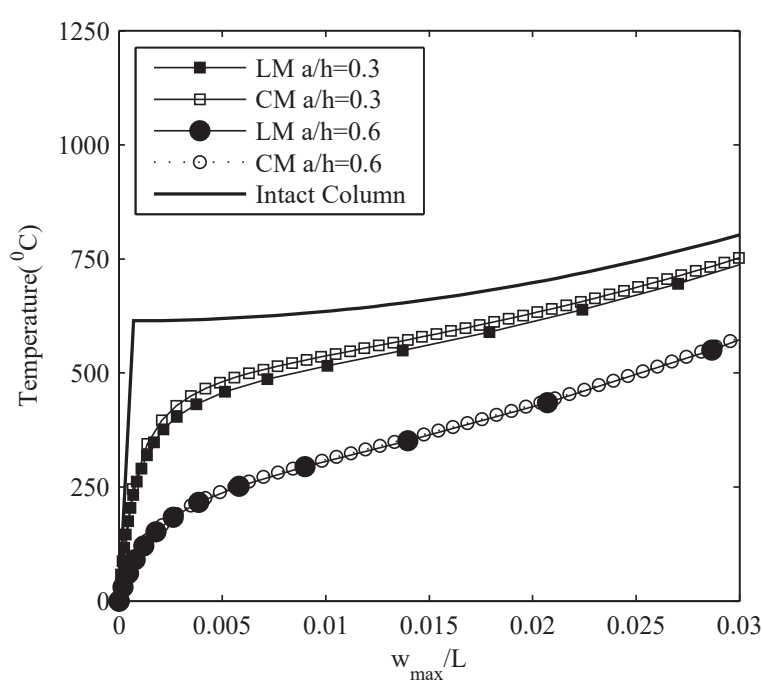

(a)

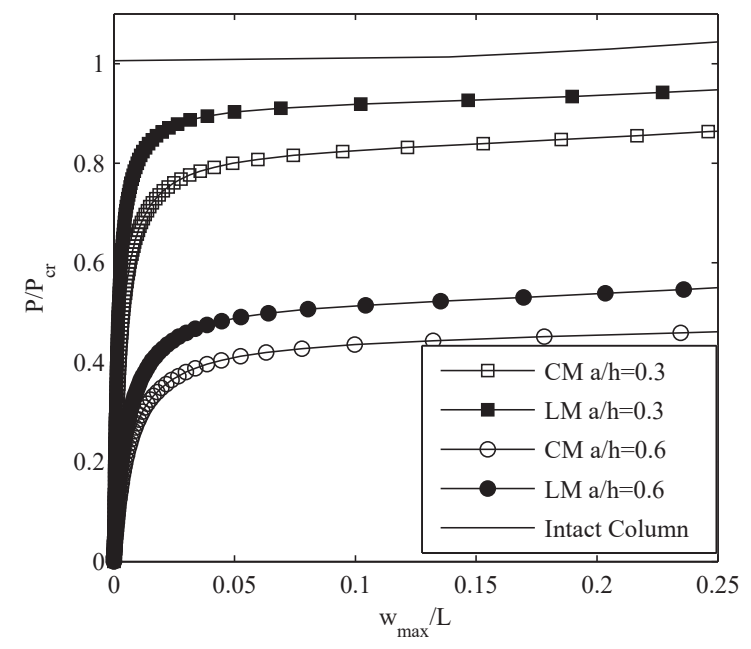

(b)

Fig. 5 Comparison results of two CM and LM techniques in postbuckling of cracked FGM beams vs. normalized maximum deflection in C-C B.Cs with different crack depths ${ }^{E_{2} / E_{1}}=0.2,{ }^{X_{0}} L=0.5$ :

a) uniform thermal loading, b) mechanical loading

- Complexity of the non-linear problem

- Modelling of the crack through the rotational spring

- Loading conditions (Mechanical load, uniform and linear thermal loads)

- Interaction between the equivalent spring stiffness factor and the non-linear part of continuity conditions.

Fig. 6 shows the effect of Young's modulus ratio on the mechanical post-buckling behavior of cracked FGM beam for SS-SS and C-C boundary conditions. A similar trend is seen in two boundary conditions as illustrated in Figs. 6(a) and 6 (b). The critical buckling load used to normalize the force axis in this figure is determined according to the critical buckling load of the intact column of the pure metal. The comparison of curves shows that the buckling 


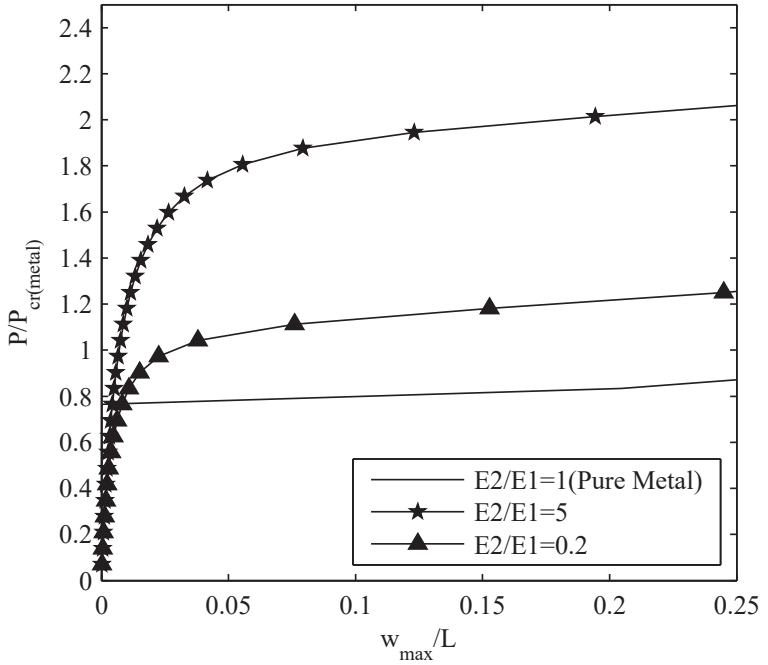

(a)

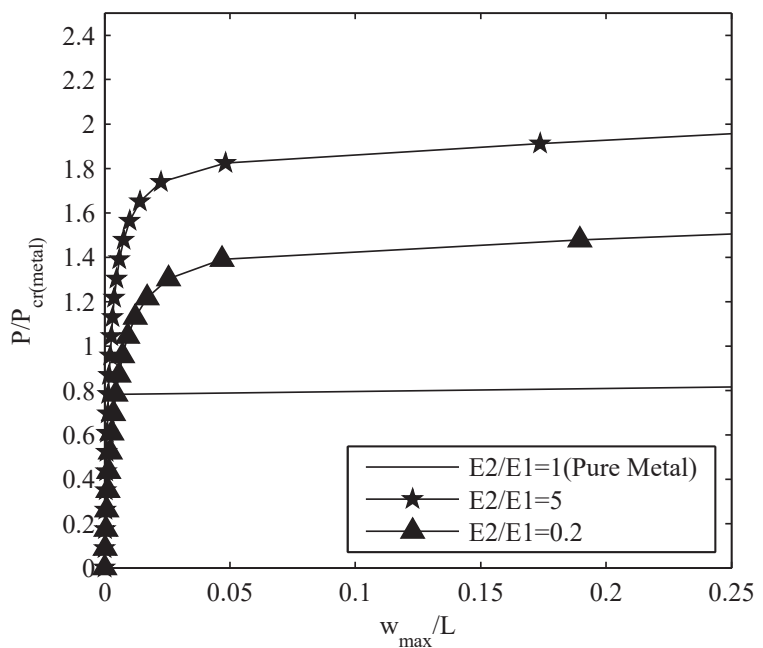

(b)

Fig. 6 Effect of Young's modulus ratio on mechanical post-buckling behavior with ${ }^{a} / h=0.5, X_{0 /}=0.5$ : a) SS-SS, b) C-C B.Cs

load capacity increases when a metal column is replaced by FGM one (in which $E_{2} / E_{1}=0.2$ with $E_{c}=E_{1}, E_{m}=E_{2}$ ). According to these curves, the buckling load capacity in $E_{2} / E_{1}=5$ increases in comparison with $E_{2} / E_{1}=0.2$. The reason for this is that the crack locates in the metal side when $E_{2} / E_{1}=5$ in which the higher value is obtained for the stiffness factor of the rotational spring.

Fig. 7 illustrates the post-buckling behavior of the SS-SS cracked FGM column with $E_{2} / E_{1}=0.2$ in different crack depths in which the influence of uniform thermal and mechanical loads is investigated in Figs. 7(a) and (b), respectively.

According to Fig. 7, the crack decreases the buckling load capacity. In Fig. 7(a), the post-buckling curve is plotted to evaluate the crack depth effect. It shows that the crack depth of 0.6 decreases the buckling load capacity more than

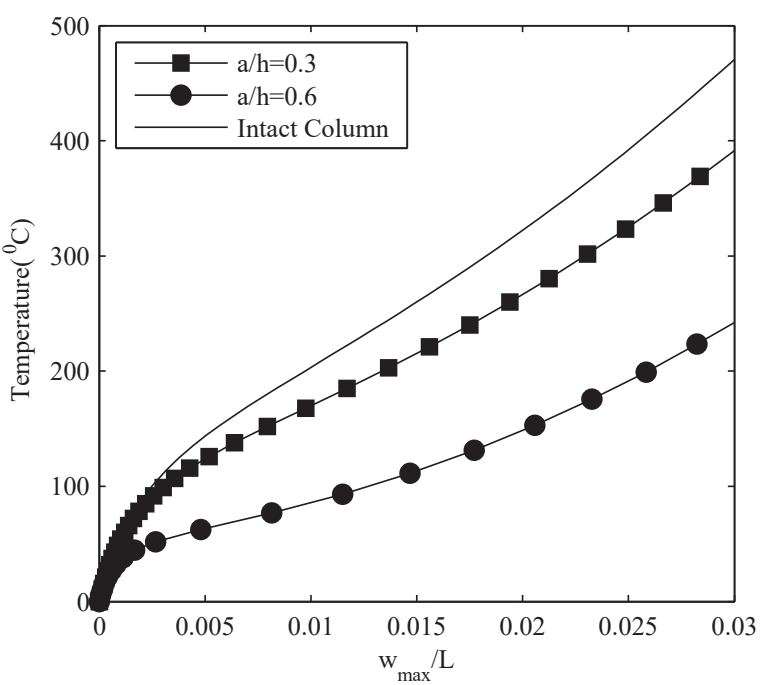

(a)

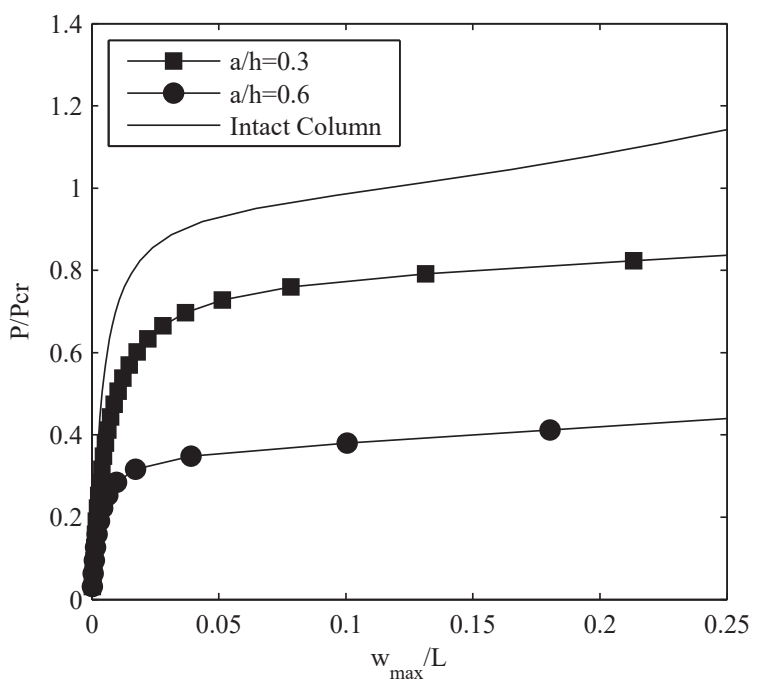

(b)

Fig. 7 Effect of crack depth on the post-buckling path of SS-SS FGM column for ${ }^{2 /} E_{1}=0.2$ under: a) uniform thermal loading, b) mechanical loading

$50 \%$. An investigation in Fig. 7(b) yields similar results in which the decrease of the buckling load capacity nearly $18 \%$ and $60 \%$ is observed for crack depths of 0.3 and 0.6 , respectively. As shown in Figs. 7(a) and 7(b), increasing the crack depth from 0.3 to 0.6 leads to the significant reduction of the buckling load capacity in comparison with the case that the crack depth increases from 0 to 0.3 . This point is due to the nonlinear relationship between the crack depth and the critical buckling load.

A comparison between Fig. 7(a) and Fig. 8(a) shows that the uniform temperature distribution makes more deformation than the linear one. The main reason for this is that all points of the column in the uniform distribution reach the same temperature (maximum), unlike the linear type. 


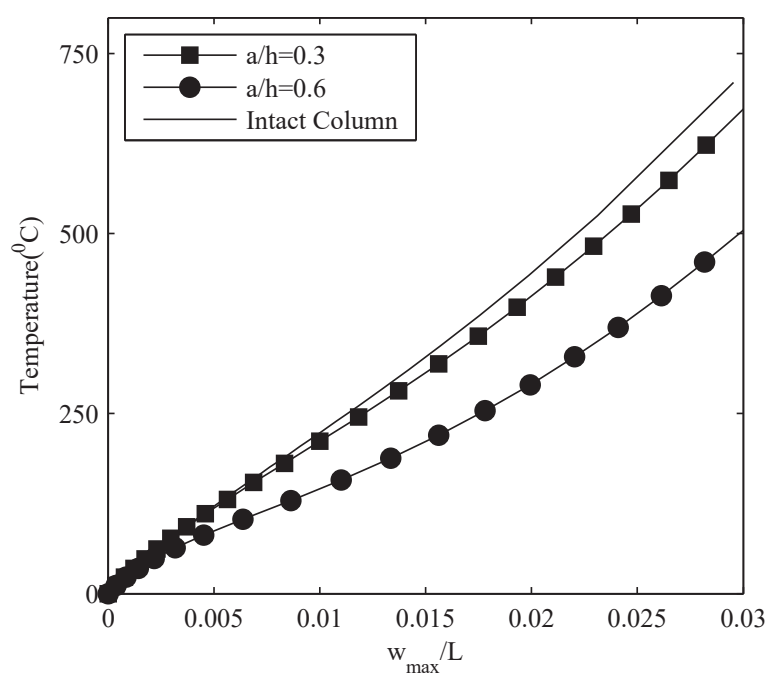

(a)

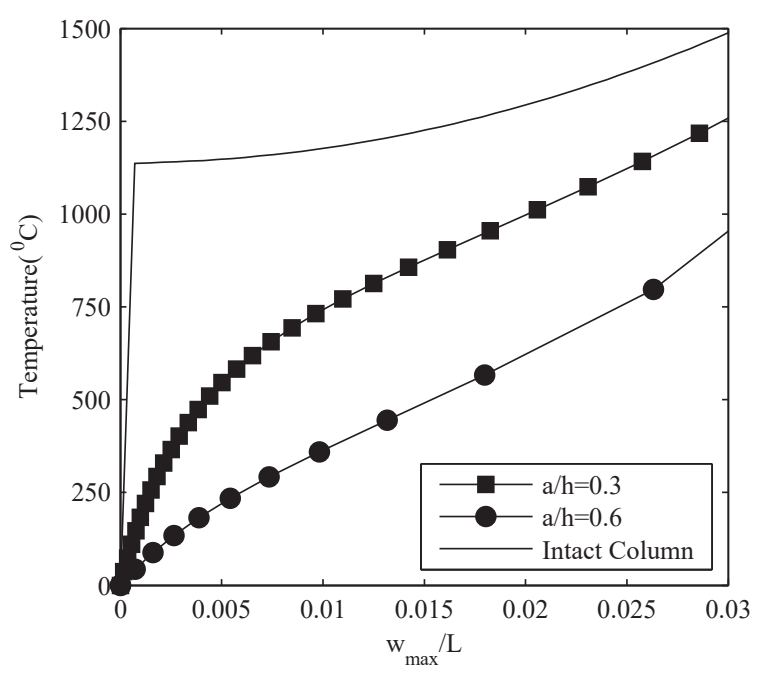

(b)

Fig. 8 Thermal post-buckling of FGM column with linear temperature distribution in different crack depths and $E_{2 /} E_{1}=0.2$ for: a) SS-SS, b) C-C B.Sc

Fig. 8 shows that a certain critical buckling point for the FGM column can be found when the column is assumed intact with $\mathrm{C}-\mathrm{C}$ end conditions. Also, a lateral deformation without any perturbation occurs in FGM columns due to the presence of the crack and stretching-bending coupling stiffness in inhomogeneous materials.

Figs. 9 and 10 depict the effect of Young's modulus ratio on the thermal post-buckling behavior of SS-SS and C-C cracked FGM columns under thermal loading with uniform and linear temperature distribution, respectively. Graphs show that the minimum to maximum thermal buckling load-bearing capacity belongs to pure metal, $E_{2} / E_{1}=0.2, E_{2} / E_{1}=5$ and pure ceramic, respectively. Furthermore, the load-bearing capacity of $\mathrm{C}-\mathrm{C}$ end conditions is nearly two times more than SS-SS. In the uniform

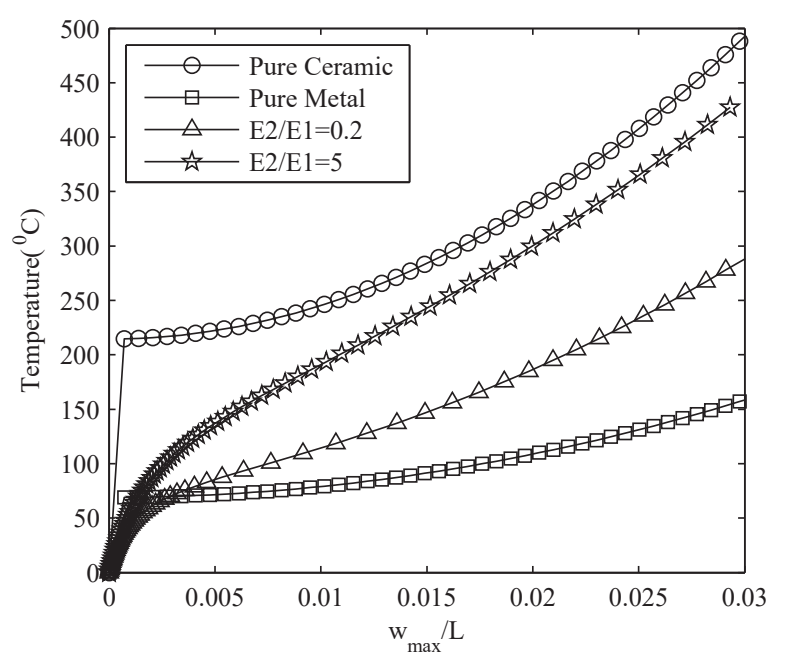

(a)

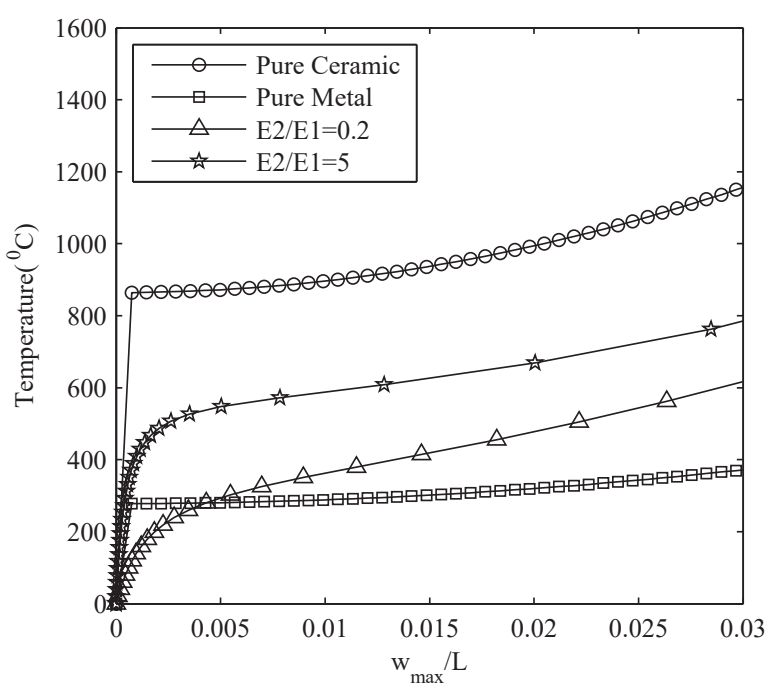

(b)

Fig. 9 Effect of Young's modulus ratio in post-buckling behavior of cracked FGM column with uniform thermal loading condition for $a_{h}=0.5, X_{0 /}=0.5:$ a) SS-SS, b) C-C B.Cs

temperature distribution as shown in Fig. 9, a certain critical buckling point can be detected for cracked isotropic columns. On the other hand, based on Figs. 9 and 10 (i.e., two types of thermal loading), a specified critical buckling load cannot be seen in cracked FGM columns. In addition, there is no obvious critical buckling load in the linear temperature distribution for all cracked columns as illustrated in Figs. 8 and 10.

\section{Conclusions}

Two techniques called LM and CM were represented to involve crack characteristics into relations of the FGM Euler-Bernoulli beam. They were utilized in the post-buckling analysis of cracked FGM columns. Moreover, their advantages and disadvantages were compared from two 


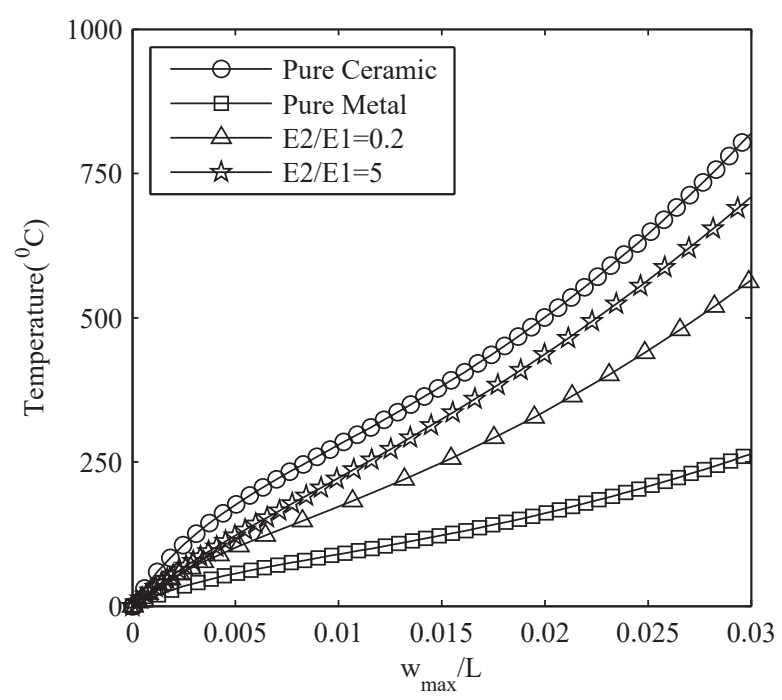

(a)

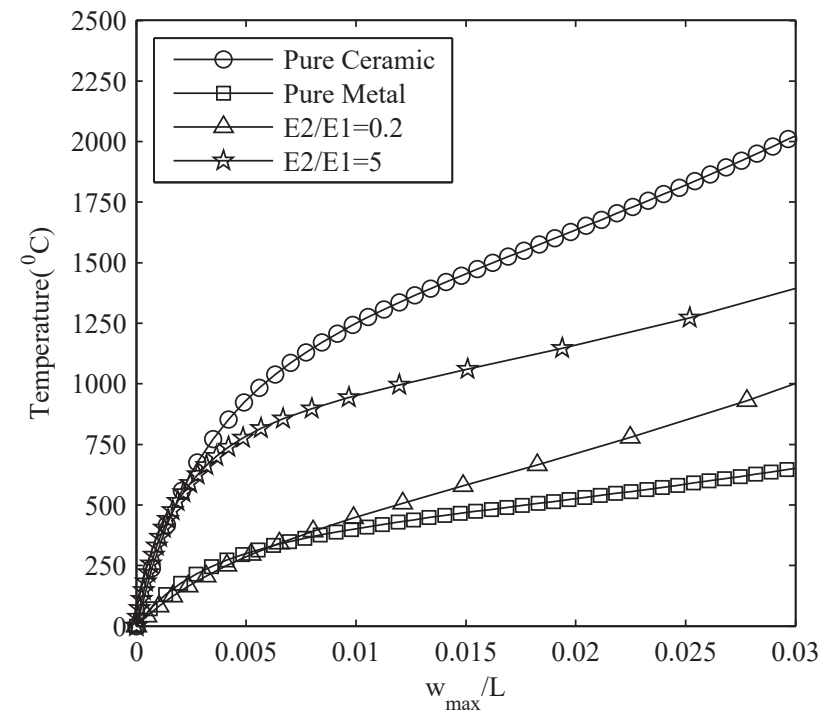

(b)

Fig. 10 Effect of material properties in post-buckling behavior of cracked FGM column with linear thermal loading condition for $a_{/ h}=0.5,{ }^{X_{0}} L=0.5:$ a) SS-SS, b) C-C B.Cs

aspects of relations and results. These techniques were developed into the framework of the FEM to derive the enriched secant and tangent stiffness matrices. Due to the restriction of the CM technique in the fulfillment of continuity conditions, the LM technique is proposed. This restriction is related to geometrical nonlinearity and material inhomogeneity equations. The LM technique

\section{References}

[1] Irwin, G. R. "Analysis of Stresses and Strains Near the End of a Crack Taversing a Plate", Transaction of ASME, Journal of Applied Mechanics, 24, pp. 361-364, 1957.

[2] Ricci, P., Viola, E. "Stress intensity factors for cracked T-sections and dynamic behaviour of T-beams", Engineering Fracture Mechanics, 73(1), pp. 91-111, 2006.

https://doi.org/10.1016/j.engfracmech.2005.06.003

[3] Yokoyama, T., Chen, M.-C. "Vibration analysis of edge-cracked beams using a line-spring model", Engineering Fracture Mechanics, 59(3), pp. 403-409, 1998.

https://doi.org/10.1016/S0013-7944(97)80283-4

[4] Mazaheri, H., Rahami, H., Kheyroddin, A. "Static and Dynamic Analysis of Cracked Concrete Beams Using Experimental Study and Finite Element Analysis", Periodica Polytechnica Civil Engineering, 62(2), pp. 337-345, 2018.

https://doi.org/10.3311/PPci.11450

[5] Okamura, H., Liu, H. W., Chu, C.-S., Liebowitz, H. "A cracked column under compression", Engineering Fracture Mechanics, 1, pp. 547-564, 1969.

https://doi.org/10.1016/0013-7944(69)90011-3

[6] Skrinar, M. "On the Application of the Simplified Crack Model in the Bending, Free Vibration and Buckling Analysis of Beams with Linear Variation of Widths", Periodica Polytechnica Civil Engineering, 63(2), pp. 423-431, 2019. https://doi.org/10.3311/PPci.12698 enables that these equations are imposed in the analysis of the cracked element. The LM implementation leads to the increase of degrees of freedom and the singularity in the matrices of the cracked element. The LM technique, despite a little more complexity, can be finely developed in various engineering problems due to flexibility and comprehensiveness.

[7] Biondi, B., Caddemi, S. "Closed form solutions of Euler-Bernoulli beams with singularities", International Journal of Solids and Structures, 42 (9-10), pp. 3027-3044, 2005.

https://doi.org/10.1016/j.ijsolstr.2004.09.048

[8] Mottaghian, F., Darvizeh, A., Alijani, A. "Extended Finite Element Method for Statics and Vibration Analyses on Cracked Bars and Beams", Journal of Solid Mechanics, 10(4), pp. 902-928, 2018. [online] Available at: http://jsm.iau-arak.ac.ir/article 545728.html

[9] Chen, W.-R., Chun, S.-C., Chang, H. "Thermal Buckling Analysis of Functionally Graded Euler-Bernoulli Beams with Temperaturedependent Properties", Journal of Applied and Computational Mechanics, 6(3), pp. 457-470, 2020.

https://doi.org/10.22055/jacm.2019.30449.1734

[10] Nasirzadeh, R., Behjat, B., Kharazi, M. "Finite element study on thermal buckling of functionally graded piezoelectric beams considering inverse effects", Journal of Theoretical and Applied Mechanics, 56(4), pp. 1097-1108, 2018. https://doi.org/10.15632/jtam-pl.56.4.1097

[11] Darvizeh, M., Darvizeh, A., Ansari, R., Alijani, A. "Pre- and post-buckling analysis of functionally graded beams subjected to statically mechanical and thermal loads", Scientia Iranica, 22(3), pp. 778-791, 2015. [online] Available at: http://scientiairanica.sharif. edu/article_3671.html 
[12] Ma, L. S., Lee, D. W. "Exact solutions for nonlinear static responses of a shear deformable FGM beam under an in-plane thermal loading", European Journal of Mechanics-A/Solids, 31(1), pp. 13-20, 2012.

https://doi.org/10.1016/j.euromechsol.2011.06.016

[13] Paul, A., Das, D. "Non-linear thermal post-buckling analysis of FGM Timoshenko beam under non-uniform temperature rise across thickness", Engineering Science and Technology, an International Journal, 19(3), pp. 1608-1625, 2016. https://doi.org/10.1016/j.jestch.2016.05.014

[14] Ke, L.-L, Yang, J., Kitipornchai, S., Xiang, Y. "Flexural Vibration and Elastic Buckling of a Cracked Timoshenko Beam Made of Functionally Graded Materials", Mechanics of Advanced Materials and Structures, 16(6), pp. 488-502, 2009. https://doi.org/10.1080/15376490902781175

[15] Song, M., Chen, L., Yang, J., Zhu, W., Kitipornchai, S. "Thermal buckling and postbuckling of edge-cracked functionally graded multilayer graphene nanocomposite beams on an elastic foundation", International Journal of Mechanical Sciences, 161-162, Article number: 105040, 2019. https://doi.org/10.1016/j.ijmecsci.2019.105040

[16] Lei, J., He, Y., Li, Z., Guo, S., Liu, D. "Postbuckling analysis of bi-directional functionally graded imperfect beams based on a novel third-order shear deformation theory", Composite Structures, 209, pp. 811-829, 2019.

https://doi.org/10.1016/j.compstruct.2018.10.106

[17] Abraham, O. N. L., Brandon, J. A. "The Modelling of the Opening and Closure of a Crack", Journal of Vibration and Acoustics, 117(3A), pp. 370-377, 1995. https://doi.org/10.1115/1.2874463

[18] Bordas, S., Rabczuk, T., Zi, G. "Three-dimensional crack initiation, propagation, branching and junction in non-linear materials by an extended meshfree method without asymptotic enrichment", Engineering Fracture Mechanics, 75(5), pp. 943-960, 2008. https://doi.org/10.1016/j.engfracmech.2007.05.010

[19] Sun, B., Huang, X., Zheng, Y., Guo, L. "Multi-scale lattice method for mesoscopic crack growth simulation of concrete structures", Theoretical and Applied Fracture Mechanics, 106, Article number: $102475,2020$.

https://doi.org/10.1016/j.tafmec.2020.102475

[20] Bruno, D., Greco, F., Lonetti, P. "A coupled interface-multilayer approach for mixed mode delamination and contact analysis in laminated composites", International Journal of Solids and Structures, 40(26), pp. 7245-7268, 2003.

https://doi.org/10.1016/j.ijsolstr.2003.09.006

[21] Liu, Z., Zheng, H., Sun, C. "A domain decomposition based method for two-dimensional linear elastic fractures", Engineering Analysis with Boundary Elements, 66, pp. 34-48, 2016. https://doi.org/10.1016/j.enganabound.2016.01.015

[22] Luciano, R., Caporale, A., Darban, H., Bartolomeo, C. "Variational approaches for bending and buckling of non-local stress-driven Timoshenko nano-beams for smart materials", Mechanics Research Communications, 103, Article number: 103470, 2020. https://doi.org/10.1016/j.mechrescom.2019.103470
[23] Alijani, A., Abadi, M. M., Darvizeh, A., Abadi, M. K. "Theoretical approaches for bending analysis of founded Euler-Bernoulli cracked beams", Archive of Applied Mechanics, 88, pp. 875-895, 2018. https://oi.org/10.1007/s00419-018-1347-0

[24] Mottaghian, F., Darvizeh, A., Alijani, A. "A novel finite element model for large deformation analysis of cracked beams using classical and continuum-based approaches", Archive of Applied Mechanics, 89, pp. 195-230, 2019. https://doi.org/10.1007/s00419-018-1460-0

[25] Alijani, A., Abadi, M. K., Razzaghi, J., Jamali, A. "Numerical analysis of natural frequency and stress intensity factor in Euler-Bernoulli cracked beam", Acta Mechanica, 230, pp. 4391-4415, 2019. https://doi.org/10.1007/s00707-019-02492-X

[26] Liew, K. M., Zhao, X., Lee, Y. Y. "Postbuckling responses of functionally graded cylindrical shells under axial compression and thermal loads", Composites Part B: Engineering, 43(3), pp. 1621-1630, 2012.

https://doi.org/10.1016/j.compositesb.2011.06.004

[27] Broek, D. "Elementary Engineering Fracture Mechanics", Springer, Dordrecht, The Netherlands, 1986. https://doi.org/10.1007/978-94-009-4333-9

[28] Ke, L.-L, Yang, J., Kitipornchai, S. "Postbuckling analysis of edge cracked functionally graded Timoshenko beams under end shortening", Composite Structures, 90(2), pp. 152-160, 2009. https://doi.org/10.1016/j.compstruct.2009.03.003

[29] Erdogan, F., Wu, B. H. "The Surface Crack Problem for a Plate With Functionally Graded Properties", Journal of Applied Mechanics, 64(3), pp. 449-456, 1997. https://doi.org/10.1115/1.2788914

[30] Wood, R. D., Schrefler, B. "Geometrically non-linear analysis A correlation of finite element notations", International Journal for Numerical Methods in Engineering, 12(4), pp. 635-642, 1978. https://doi.org/10.1002/nme.1620120408

[31] Panda, S. K., Singh, B. N. "Thermal post-buckling analysis of a laminated composite spherical shell panel embedded with shape memory alloy fibres using non-linear finite element method", Proceedings of the Institution of Mechanical Engineers, Part C: Journal of Mechanical Engineering Science, 224(4), pp. 757-769, 2010.

https://doi.org/10.1243/09544062JMES1809

[32] Alijani, A., Darvizeh, M., Darvizeh, A., Ansari, R. "On nonlinear thermal buckling analysis of cylindrical shells", Thin-Walled Structures, 95, pp. 170-182, 2015. https://doi.org/10.1016/j.tws.2015.06.013

[33] Wagner, W., Wriggers, P. "A simple method for the calculation of postcritical branches", Engineering Computations, 5(2), pp. 103109, 1988. https://doi.org/10.1108/eb023727

[34] Wriggers, P. "Nonlinear Finite Element Methods", Springer, Berlin, Germany, 2008. https://doi.org/10.1007/978-3-540-71001-1

[35] Ganesan, N., Kadoli, R. "Buckling and dynamic analysis of piezothermoelastic composite cylindrical shell", Composite Structures, 59(1), pp. 45-60, 2003. https://doi.org/10.1016/S0263-8223(02)00230-1 


\section{Appendix A}

The expanded form of Eqs. (42) to (44)

$$
\begin{aligned}
& \frac{6 \hat{D} b}{K_{t} x_{0}{ }^{2}} w_{2}-\frac{\hat{B} b}{2 K_{t}} \phi_{2}{ }^{2}-\frac{4 \hat{D} b}{K_{t} x_{0}} \phi_{2}-\phi_{3} \\
= & \frac{\hat{B} b}{K_{t} l e} u_{1}+\frac{6 \hat{D} b}{K_{t} x_{0}{ }^{2}} w_{1}+\frac{2 \hat{D} b}{K_{t} x_{0}} \phi_{1}-\frac{\hat{B} b}{K_{t} l e} u_{4} \\
& \left.\frac{6 \hat{D} b}{x_{0}^{2}}-\frac{6 \hat{D} b}{\left(l e-x_{0}\right)^{2}}\right) w_{2}+\frac{\hat{B} b}{2} \phi_{2}{ }^{2}-\frac{4 \hat{D} b}{x_{0}} \phi_{2}-\frac{4 \hat{D} b}{\left(l e-x_{0}\right)} \phi_{3}-\frac{\hat{B} b}{2} \phi_{3}{ }^{2} \\
= & \frac{6 \hat{D} b}{x_{0}{ }^{2}} w_{1}+\frac{2 \hat{D} b}{x_{0}} \phi_{1}-\frac{6 \hat{D} b}{\left(l e-x_{0}\right)^{2}} w_{4}+\frac{2 \hat{D} b}{\left(l e-x_{0}\right)} \phi_{4} \\
& \frac{6 \hat{B} b}{x_{0}^{2}}\left(w_{1} \phi_{2}-w_{2} \phi_{2}\right)+\frac{2 \hat{B} b}{x_{0}}\left(\phi_{1} \phi_{2}+2 \phi_{2}^{2}\right) \\
- & \frac{6 \hat{D} b}{x_{0}^{2}}\left(\phi_{1}+\phi_{2}\right)+\frac{12 \hat{D} b}{x_{0}^{3}}\left(w_{2}-w_{1}\right) \\
= & \frac{6 \hat{B} b}{\left(l e-x_{0}\right)^{2}}\left(w_{4} \phi_{3}-w_{2} \phi_{3}\right)-\frac{2 \hat{B} b}{\left(l e-x_{0}\right)}\left(\phi_{3} \phi_{4}+2 \phi_{3}^{2}\right) \\
- & \frac{12 \hat{D} b}{\left(l e-x_{0}\right)^{3}}\left(w_{2}+w_{4}\right)-\frac{6 \hat{D} b}{\left(l e-x_{0}\right)^{2}}\left(\phi_{3}+\phi_{4}\right)
\end{aligned}
$$

\section{Appendix B}

Matrices mentioned in Eq. (61) for a cracked element are symmetric with dimension $(17 \times 17)$. Non-zero components of these matrices are represented as follows

$$
\begin{aligned}
& \boldsymbol{K}_{\text {spring }(6,6)}=K_{t}, \boldsymbol{K}_{\text {spring }(6,14)}=-K_{t}, \boldsymbol{K}_{T 1(4,7)}=1, \\
& \boldsymbol{K}_{T 1(7,12)}=-1, \boldsymbol{K}_{T 2(5,8)}=1, \boldsymbol{K}_{T 2(8,13)}=-1, \\
& \boldsymbol{K}_{T 3(1,9)}=d_{3}, \boldsymbol{K}_{T 3(2,9)}=e_{3}, \boldsymbol{K}_{T 3(3,9)}=f_{3}, \\
& \boldsymbol{K}_{T 3(5,9)}=a_{3}, \boldsymbol{K}_{T 3(6,6)}=2 \lambda_{3} b_{3}, \boldsymbol{K}_{T 3(6,9)}=2 b_{3} \phi_{2}+c_{3}, \\
& \boldsymbol{K}_{T 3(9,14)}=-1, \boldsymbol{K}_{T 3(9,15)}=g_{3}, \boldsymbol{K}_{T 4(2,10)}=f_{4}, \\
& \boldsymbol{K}_{T 4(3,10)}=g_{4}, \boldsymbol{K}_{T 4(5,10)}=a_{4}, \boldsymbol{K}_{T 4(6,6)}=2 \lambda_{4} b_{4}, \\
& \boldsymbol{K}_{T 4(6,10)}=2 b_{4} \phi_{2}+c_{4}, \boldsymbol{K}_{T 4(10,14)}=2 e_{4} \phi_{3}+d_{4}, \\
& \boldsymbol{K}_{T 4(10,16)}=h_{4}, \boldsymbol{K}_{T 4(10,17)}=r_{4}, \boldsymbol{K}_{T 4(14,14)}=2 \lambda_{4} e_{4}, \\
& \boldsymbol{K}_{T 5(2,6)}=\lambda_{5} a_{5}, \boldsymbol{K}_{T 5(3,6)}=\lambda_{5} b_{5}, \boldsymbol{K}_{T 5(5,6)}=\lambda_{5} c_{5}, \\
& \boldsymbol{K}_{T 5(6,6)}=\lambda_{5} d_{5}, \boldsymbol{K}_{T 5(2,11)}=\left(a_{5} \phi_{2}+e_{5}\right), \boldsymbol{K}_{T 5(3,11)}=\left(b_{5} \phi_{2}+f_{5}\right) \\
& \boldsymbol{K}_{T 5(5,11)}=\left(c_{5} \phi_{2}+k_{5} \phi_{3}+g_{5}+s_{5}\right), \boldsymbol{K}_{T 5(5,14)}=\lambda_{5} k_{5}, \\
& \boldsymbol{K}_{T 5(6,11)}=a_{5} w_{1}+b_{5} \phi_{1}+c_{5} w_{2}+2 d_{5} \phi_{2}+h_{5}, \\
& \boldsymbol{K}_{T 5(11,14)}=2 l_{5} \phi_{3}+y_{5}+k_{5} w_{2}+m_{5} w_{4+} n_{5} \phi_{4}, \\
& \boldsymbol{K}_{T 5(11,16)}=r_{5}+m_{5} \phi_{3}, \boldsymbol{K}_{T 5(11,17)}=t_{5}+n_{5} \phi_{3}, \\
& \boldsymbol{K}_{T 5(14,14)}=2 \lambda_{5} l_{4}, \boldsymbol{K}_{T 5(14,16)}=\lambda_{5} m_{5}, \boldsymbol{K}_{T 5(14,17)}=\lambda_{5} n_{5}
\end{aligned}
$$

also $\boldsymbol{K}_{T L}$ and $\boldsymbol{K}_{T R}$ are

$$
\begin{aligned}
\boldsymbol{K}_{T L} & =\left[\begin{array}{cc}
\text { Eq. }(14)_{6 \times 6} & 0 \\
0 & 0
\end{array}\right]_{17 \times 17} \\
\boldsymbol{K}_{T R} & =\left[\begin{array}{cc}
0 & 0 \\
0 & \text { Eq. }(14)_{6 \times 6}
\end{array}\right]_{17 \times 17}
\end{aligned}
$$

\section{Nomenclature}

a

$b$

$\boldsymbol{C}_{L(R)} \quad$ conversion matrix of Left (right) sub-element

$\boldsymbol{C}_{\text {Spring }}$ conversion matrix related to rotational spring

$E_{0} \quad$ FGM Young's modulus in middle plane

$E_{1} \quad$ FGM Young's modulus of crack side

$E_{2} \quad$ FGM Young's modulus of intact side

$E_{c} \quad$ Young modulus of ceramic

$E_{m} \quad$ Young modulus of metal

$\boldsymbol{F}_{\text {ext }} \quad$ external force vector

$\overline{\boldsymbol{F}}_{\text {th }} \quad$ thermal force vector

$\hat{G} \quad$ flexibility due to crack

$h \quad$ height

$H_{i} \quad i$ th constraint related to continuity condition

$k_{I} \quad$ stress intensity factor of crack opening mode

$k_{T L(T R)} \quad$ tangent stiffness matrix of left (right) sub-element in terms of displacements of middle nodes

$\boldsymbol{K}_{0} \quad$ stiffness matrix of intact element

$K_{t} \quad$ spring stiffness factor of the rotational spring

$K_{\text {spring }} \quad$ stiffness matrix of rotational spring

$K_{\text {S.crack }}$ secant stiffness matrix of cracked element

$K_{t h} \quad$ thermal stiffness matrix

$K_{T} \quad$ tangent stiffness matrix

$K_{T i} \quad$ tangent stiffness matrix corresponding to ith constraint

$K_{\text {T.crack }}$ tangent stiffness matrix of cracked element

$K_{T L(T R)} \quad$ tangent stiffness matrix of left (right) sub-element in terms of displacements of basic nodes

L length

$M \quad$ bending moment

$T_{m} \quad$ metal temperature

$T_{c} \quad$ ceramic temperature 


\begin{tabular}{|c|c|}
\hline$u$ & axial displacement field \\
\hline$u_{i}$ & axial displacement of $i$ th node \\
\hline$u_{e L(e R)}$ & $\begin{array}{l}\text { axial displacement field of left (right) } \\
\text { sub-element }\end{array}$ \\
\hline$u$ & nodal displacement vector \\
\hline $\boldsymbol{u}_{L(R)}$ & $\begin{array}{l}\text { nodal displacement vector of left(right) } \\
\text { sub-element }\end{array}$ \\
\hline$U$ & strain energy \\
\hline$U_{L(R)}$ & strain energy of left (right) sub-element \\
\hline$U_{\text {Spring }}$ & strain energy of rotational spring \\
\hline$V_{e L(e R)}$ & shear force of left (right) sub-element \\
\hline$w_{i}$ & transverse displacement of ith node \\
\hline$w_{e L(e R)}^{\prime}$ & slope field of left (right) sub-element \\
\hline$x_{0}$ & crack location in element \\
\hline$X_{0}$ & crack location in column \\
\hline$\alpha_{c}$ & thermal expansion coefficient of ceramic \\
\hline$\alpha_{m}$ & thermal expansion coefficient of metal \\
\hline$\beta$ & $\begin{array}{l}\text { constant relevant to gradual variation of } \\
\text { material properties }\end{array}$ \\
\hline
\end{tabular}

$\begin{array}{ll}\varepsilon_{x} & \text { strain } \\ \varepsilon_{0} & \text { linear strain } \\ \varepsilon_{N L} & \text { non-linear strain } \\ \xi & \text { crack depth ratio } \\ \eta & \text { load level } \\ \lambda_{i} & \text { ith Lagrange multiplier } \\ v & \text { poisson's ratio } \\ \sigma_{x} & \text { stress } \\ \sigma_{0} & \text { linear stress } \\ \sigma_{N L} & \text { non-linear stress } \\ \sigma_{\text {th }} & \text { thermal stress tensor } \\ \Pi & \text { total potential energy } \\ \Pi_{\text {crack }} & \text { total potential energy of cracked element } \\ \phi_{i} & \text { slope of ith node } \\ \Omega & \text { potential energy of external forces } \\ \Omega_{\text {crack }} & \begin{array}{l}\text { potential energy of external forces for cracked } \\ \text { element }\end{array} \\ \Delta_{0} T & \begin{array}{l}\text { change of temperature relative to the reference } \\ \text { temperature }\end{array} \\ \end{array}$

